\title{
Potencial de uso das espécies arbóreas de uma floresta secundária, na Zona Bragantina, Pará, Brasil
}

\author{
Fabrízia de Oliveira ALVINO'1, Manoela Ferreira F. da SILVA², Breno Pinto RAYOL ${ }^{2}$
}

\section{RESUMO}

Este estudo teve como objetivo avaliar o potencial de uso das espécies arbóreas de uma floresta secundária com aproximadamente 30 anos de idade, abandonada após sucessivos ciclos de agricultura itinerante. A área de estudo está localizada em propriedade de agricultura familiar na Zona Bragantina ${ }_{2}$ PA. Aárea inventariada corresponde a 1,5 ha, onde foram medidos todos os espécimes arbóreos com DAP $35 \mathrm{~cm}$. Verificou-se a ocorrência de 103 espécies, que totalizaram 1961 indivíduos/ha e área basal de $17,7 \mathrm{~m}^{2} /$ ha. As espécies com maior número de indivíduos foram: Sacoglottis amazonica Mart., Ormosia flava (Ducke) Rudd, Eschweilera coriacea (DC.) S.A. Mori e Croton matourensis Aubl.. As categorias de uso que apresentam maior número de espécies foram: Construções rurais (33\%), seguida das espécies madeireiras de alto valor comercial (30\%), madeireiras de baixo valor comercial e as utilizadas como lenha ( $9 \%$ cada).

\section{PALAVRAS-CHAVE}

Potencial de uso, Espécies arbóreas, Floresta secundária.

\section{Potential of use of the tree species in a secondary forest, of the Bragantina zone, Pará, Brazil}

\begin{abstract}
The aim of this paper was to determine the economic potential of a secondary forest with about 30 years of age that was left to regrowth after successive cycles of swidden agriculture. The study area is located in a family farm in the Bragantina Zone ${ }_{2} P A$. The inventoried area was $1.5 \mathrm{ha}$, where all the tree specimens with $\mathrm{DBH}^{3} 5 \mathrm{~cm}$ were measured. A total of 103 species were identified, corresponding to 1961 individuals/ha and $17.7 \mathrm{~m}^{2} /$ ha basal area, the species with most number of individuals being Sacoglottis amazonica Mart., Ormosia flava (Ducke) Rudd, Eschweilera coriacea (DC.) S.A. Mori and Croton matourensis Aubl. The categories with bighest number of species were rural construction (33\%), followed by bigh value commercial timber species (30\%), low value commercial and firewood (9\% each).
\end{abstract}

\section{KEYWORDS}

Economic potential, Tree species, Secondary forest

\footnotetext{
${ }^{1}$ Universidade Federal Rural da Amazônia, Departamento de Ciências Florestais, Av. Tancredo Neves S/N, cx. Postal 917, 66077-530 - Belém, PA, Brasil. e-mail: fabriziaalvino@yahoo.com.br

${ }^{2}$ Museu Paraense Emílio Goeldi, Av. Magalhães Barata, 376-São Braz. 66040-170 - Belém, PA, Brasil.
} 


\section{ACTA \\ AMAZONICA}

POTENCIAL DE USO DAS ESPÉCIES ARBÓREAS DE UMA

FLORESTA SECUNDÁRIA, NA ZONA BRAGANTINA, PARÁ, BRASIL

\section{INTRODUÇÃO}

A vegetação secundária (capoeira) surge a partir do abandono da área utilizada após o desenvolvimento de atividades como a agricultura e pecuária. Estima-se na microregião Bragantina que 53\% de sua área total que teve sua floresta nativa removida está coberta por este tipo de vegetação (Alencar et al., 1996; Vieira, 1996).

AZona Bragantina, localizada no nordeste paraense, tornouse um exemplo bem conhecido de como o uso intensivo da terra pode levar a uma completa modificação na paisagem original e de suas condições ecológicas (Denich, 1986).

Rios et al. (2001) afirmam que as florestas secundárias, nas diversas etapas de desenvolvimento, são capazes de prover importantes recursos às populações tradicionais. Além disso, desempenham um papel de elevada importância ecológica, em termos de crescimento florestal, acúmulo de biomassa, controle de erosão, conservação de nutrientes, benefícios hidrológicos e manutenção da biodiversidade (Nepstad et al., 1996; Pereira \& Vieira, 2001). Nelas pode-se encontrar essências madeireiras de rápido crescimento e de boa formação com alto valor econômico (Denich, 1991).

O manejo deste tipo florestal é uma forma relativamente nova de uso alternativo da terra e ainda não foi possível descrever o sucesso prático dessa atividade, embora existam exemplos bem sucedidos de manejo de florestas secundárias na Costa Rica (Finegan, 1992).

Segundo Fachim \& Guarim (1995), o conhecimento inadequado da biologia das espécies, de seus potenciais para diferentes usos e de variabilidade genética inter e intra populacionais existentes vêm levando à perda irreversível de recursos genéticos de espécies, antes que esses estudos, ao menos, tenham sido efetuados. No entanto, há carência de estudos que visem a identificação da potencialidade de uso de espécies de floresta secundária, dificultando a possibilidade de manejá-los.

O objetivo deste trabalho foi de realizar o levantamento e a divulgação do potencial de uso das espécies arbóreas encontradas em floresta secundária, visando aumentar o conhecimento deste tipo de vegetação, dar base a outros trabalhos mais específicos, assim como fornecer subsídios ao manejo dessas áreas, a fim de que a floresta secundária possa vir a servir como uma fonte complementar de renda à agricultura familiar, no estado.

\section{MATERIAL E MÉTODOS}

Aárea estudada situa-se na comunidade Benjamin Constant, localizada no município de Bragança-PA, nas coordenadas geográficas $01^{\circ} 11^{\prime} 22^{\prime \prime}$ de latitude Sul e $46^{\circ} 40^{\prime} 41^{\prime \prime}$ de longitude Oeste (Rios et al., 2001).

O clima da região apresenta temperatura média anual de $25^{\circ}$ a $26^{\circ} \mathrm{C}$, pluviosidade de 2.200 a $3.000 \mathrm{~mm}$ por ano e umidade relativa média de $85 \%$, com solos ácidos de boa drenagem (IBGE, 1983).
A floresta secundária possui cerca de 30 anos de sucessão, pertencente a um agricultor familiar e provêm de área abandonada após sucessivos cultivos anuais de milho, mandioca e arroz.

A fim de determinar a composição, importância e uso das espécies do estrato arbóreo, foi realizado um inventário florístico/etnobotânico, por amostragem, onde foram delimitadas sistematicamente 6 parcelas de 50 x $50 \mathrm{~m}(2500$ $\mathrm{m}^{2}$ ), divididas em 25 subparcelas de $10 \times 10 \mathrm{~m}\left(100 \mathrm{~m}^{2}\right)$ cada, com área total amostral de 1,5 ha, onde foram incluídos na amostragem todos os indivíduos arbóreos vivos, com diâmetro a 1,30 m acima do solo (DAP) igual ou superior a $5 \mathrm{~cm}$. Além do diâmetro, outra variável observada foi a altura, estimada com auxílio de uma vara de dois metros. Esses dados foram anotados em fichas de campo.

O material botânico foi coletado e identificado ao nível de família, gênero e espécie por meio de comparações com exsicatas depositadas nos Herbários do Museu Paraense Emílio Goeldi-MPEG e Embrapa Amazônia Oriental, ambos localizados em Belém-PA.

Os dados foram processados e analisados pelo programa Sistema Florestal Contínuo (SFC) desenvolvido pela Embrapa Amazônia Oriental em parceria com a Universidade Federal Rural da Amazônia (UFRA).

De posse dos resultados obtidos pelo levantamento florístico realizado, foram identificadas as espécies com potencial de uso (madeireiro e não madeireiro) a serem manejadas futuramente.

Através de uma abordagem participativa entre a equipe técnica (literaturas específicas) e o agricultor (conhecimento empírico), as espécies foram separadas em oito grupos de uso: madeira de alto valor comercial, madeira de baixo valor comercial, madeira para construção rural, madeira para lenha, frutos, extrativos, medicinal e artesanal.

\section{RESULTADOS E DISCUSSÃO}

No levantamento efetuado foram amostradas 103 espécies distribuídas em 74 gêneros e 35 famílias botânicas, compondo densidade total de 1961 indivíduos/hectare (ind./ha) e área basal total de $17,7 \mathrm{~m}^{2} / \mathrm{ha}$. A tabela 1 apresenta os valores de percentagem da densidade e área basal dos indivíduos inventariados por grupo de uso.

As categorias de uso que apresentaram maior número de espécies na área estudada foram: Madeiras utilizadas em construções rurais (33\%) e de alto valor comercial (30\%), seguida pelas madeireiras de baixo valor comercial (9\%), utilizadas como lenha (9\%), frutíferas (7\%), medicinais (5\%), artesanais (4\%) e as com potencial resinífero (1\%). As espécies sem uso conhecido representaram somente $2 \%$.

Dentre o grupo de espécies no inventário florestal que podem ser utilizadas para construções rurais, destacaram-se, em termos de abundância, Maprounea guianensis (158 ind./ ha), Myrciaria tenella (127 ind./ha), Myrcia bracteata (92 ind./ ha), Casearia arborea (88 ind./ha), Phyllanthus nobilis (87 ind./ 


\section{ACTA \\ AMAZONICA}

Tabela 1 - Percentagem da densidade e área basal por grupo de uso dos indivíduos encontrados em uma floresta secundária, no município de Bragança, PA.

\begin{tabular}{|c|c|c|}
\hline Grupo de Uso & Densidade (\%) & Área basal (\%) \\
\hline $\begin{array}{l}\text { Madeira de alto } \\
\text { valor comercial }\end{array}$ & 27,4 & 31,6 \\
\hline $\begin{array}{l}\text { Madeira de baixo } \\
\text { valor comercial }\end{array}$ & 3,5 & 7,9 \\
\hline $\begin{array}{l}\text { Madeira para } \\
\text { construção rural }\end{array}$ & 47,3 & 43,0 \\
\hline $\begin{array}{l}\text { Madeira para } \\
\text { lenha }\end{array}$ & 3,9 & 2,8 \\
\hline Frutos & 6,2 & 6,7 \\
\hline Extrativos & 0,2 & 0,1 \\
\hline Medicinal & 1,2 & 1,4 \\
\hline Artesanal & 2,0 & 1,2 \\
\hline Sub-total & 91,7 & 94,7 \\
\hline $\begin{array}{l}\text { Sem uso } \\
\text { conhecido }\end{array}$ & 8,3 & 5,3 \\
\hline Total & 100,0 & 100,0 \\
\hline
\end{tabular}

ha) e Talisia longifolia (55 ind./ha) representando $66 \%$ da densidade total dos indivíduos deste grupo (anexo 1).

As espécies que apresentaram maiores valores de densidade na categoria de madeiras de alto valor comercial, foram Sacoglottis amazonica (129 ind./ha) seguida de Ormosia flava (119 ind./ha) e Eschweilera coriacea (77 ind./ha), Croton matourensis (57 ind./ha),Lecythis lurida (31 ind./ha) e Lecythis pisonis (22 ind./ha) (anexo 1).

Espécies florestais de valor econômico como o jatobá (Hymenaea courbaril), ipê-amarelo (Tabebuia serratifolia), louro-prata (Ocotea guianensis), tauarí (Couratari guianensis), sucupira-amarela (Diplotropispurpurea), paricá (Schizolobium amazonicum) possuíam poucos indivíduos: 4, 3, 3, 2, 1 e 1 respectivamente. A retirada de espécies para extração de madeira, com baixas densidades ou, segundo Fachim \& Guarim (1995), exclusivas de algum tipo de solo ou formação vegetal, sem que tenham a possibilidade de completarem o seu ciclo reprodutivo, leva ao desaparecimento dessas espécies. Portanto, em caso de corte esses indivíduos devem ser preservados da exploração, para virem servir como árvores matrizes, mantendo assim a diversidade, riqueza e perpetuação das espécies na área.

Lisboa (1989) ao realizar um estudo florístico em 0,5 hectare de floresta secundária de aproximadamente 15 anos, na BR364 (Cuiabá-Porto Velho), estado de Rondônia, comenta que a constituição florística mostra um povoamento com espécies que podem ser aproveitadas economicamente pelos seus colonos, se estes conhecessem os potenciais de utilização das espécies, porém complementa que muitas espécies, como Acacia polyphylla, Bagassa guianensis, Caryocar glabrum, Cedrela odorata, Couratari guianensis, Schizolobium amazonicum etc., já são de utilização conhecida na construção civil e naval. O paricá,Schizolobium amazonicum, por exemplo, tem sido muito explorado, tanto em áreas de vegetação natural quanto em áreas abandonadas, como matéria prima para a indústria de compensados e a sua exploração na capoeira é possível, levando-se em consideração suas altas taxas de regeneração e seu crescimento rápido.

Em relação à área basal, fator de elevada importância, quando se refere a produtos florestais madeireiros, a espécie que mais se destacou no grupo de madeira de alto valor comercial foi Croton matourensis. Com $1,5 \mathrm{~m}^{2}$ por hectare, esta espécie apresentou poucos indivíduos, porém de grande porte. Já a espécie Sacoglottis amazonica, apresentou grande quantidade de indivíduos de médio porte, o que lhe fez ocupar a segunda posição com $1,1 \mathrm{~m}^{2} /$ ha.

Vieira et al. (1996), além de caracterizarem morfologicamente frutos e sementes de espécies arbóreas da Amazônia, incluíram nos seus estudos os usos econômicos e os dispersores das mesmas. Entre elas estavam: Sacoglottis amazonica, Eschweilera coriacea e Dipteryx odorata que foram classificadas como espécies madeireiras, e dispersas por mamíferos.

As espécies Tapirira guianensis (46 ind./ha), Licania densiflora (12 ind./ha) e Sclerolobium paraense (5 ind./ha) representaram $90 \%$ da densidade total das espécies de madeira de baixo valor comercial. As espécies acima citadas continuam nas mesmas posições quando refere-se à área basal, apresentando $11,0,2 \mathrm{e} 0,2 \mathrm{~m}^{2}$ por hectare, respectivamente.

Dados sobre o processamento, durabilidade natural e indicação de uso de algumas espécies são encontrados no levantamento do potencial madeireiro na área do Grande Carajás, realizado pelo IBDF (1983). Dentre elas pode-se citar Tapirira guianensis, que apresentou secagem artificial rápida com fortes rachaduras e empenamentos, fácil trababilidade e pouca resistência ao ataque de insetos e intempéries, sendo indicada para acabamentos, marcenaria, laminados e compensados.

Entre as espécies que possuem potencial de uso para lenha, as mais representativas em termos de abundância foram Abarema jupunba (39 ind./ha), Inga rubiginosa (13 ind./ha) e Inga paraensis ( 7 ind./ha), perfazendo um total de $77 \%$ da densidade total desse grupo de uso.

As espécies Annona paludosa com 71 ind./ha, Platonia insignis com 24 ind./ha, Psidium guajava, 12 ind./ha, Inga edulis e Inga nobilis com 5 ind./ha cada, Inga macrophylla $\mathrm{e}$ Byrsonima aerugo com 2 ind./ha, foram enquadradas na seção das plantas que apresentam frutos comestíveis.

A espécie Annona paludosa, conhecida como envira-biribá, foi uma das 95 espécies ilustradas e descritas por Rocha e Silva (2002) em um catálogo de espécies de floresta secundária do nordeste paraense. Estes autores confirmam que Annona paludosa produz fruto comestível e madeira leve freqüentemente utilizada como cabo de ferramenta.

O bacurizeiro, Platonia insignis, ocorre naturalmente em florestas secundárias em diversos estádios de sucessão (Rios 
et al., 2001) e em áreas degradadas e arenosas, sendo indiferente à textura e fertilidade do solo (Shanley et al., 1998). Sua polpa é doce, muito agradável e vem sendo utilizada na fabricação de cremes, sucos e xaropes. Também pode ser consumida in natura (Rios et al., 2001).

O bacurí é uma das frutas mais populares em Belém (Shanley et al.,1998). Ccerca de sete milhões de frutas foram vendidas nas principais feiras da capital do Pará em 1994, gerando cerca de 1,6 milhões de reais.

Clement \& Arkcoll (1979) mencionam que a diversidade de espécies e variedades frutíferas não é grande somente em números de frutos, mas também nas características importantes em produtos que podem ser processados e desenvolvidos. Cavalcante (1979) realizou um levantamento de 42 espécies frutíferas cultivadas e nativas da região amazônica, com o objetivo de divulgar as frutas comestíveis e seu elevado potencial econômico. Dentre as espécies citadas pelo autor, encontra-se Inga macrophylla, que apresenta frutos de até $35 \mathrm{~cm}$ de comprimento e $5 \mathrm{~cm}$ de largura, casca revestida de pêlos amarelos, levemente ásperos. Suas sementes encontram-se envolvidas por abundante polpa branca, adocicada, de sabor regular e comestível.

Ferreira (2000), ao identificar as plantas medicinais utilizadas por uma comunidade pesqueira do litoral paraense, observou que florestas secundárias em estágios de sucessão mais avançados, são freqüentemente visitadas pelos moradores. Além de proporcionarem madeiras para produção de carvão e construção de casas, fornecem frutos e plantas medicinais. Neste mesmo estudo, Himatanthus sucuuba foi a espécie que obteve maior freqüência de citações, utilizando-se o látex para tosse, por meio da ingestão do leite, e para dores no corpo por meio de implasto. Outra parte freqüentemente utilizada é a casca, no combate à ulcera e inflamações no útero. Vieira (1991) comenta que essa planta é usada popularmente como vermífugo e contra micoses, porém adverte que o uso caseiro desta planta normalmente não é aconselhável, devido conter princípios ativos tóxicos.

No presente estudo, Himatanthus sucuuba obteve 21 indivíduos por hectare, representando $89 \%$ da densidade total das espécies com potencial de utilização medicinal. As demais espécies: Stryphnodendron adstringens, Siparuna guianensis, Clavija lancifolia e Luehea sp. apresentaram somente um único indivíduo em toda a área.

Segundo Vieira (1991), a casca de Stryphnodendron adstringens (barbatimão) possui alto poder adstringente, podendo ser utilizada externamente na forma de pó para o tratamento de úlceras, ou em banhos contra leucorréia, catarros uretral e vaginal e internamente, utilizada em decoto para gastrite e no controle do câncer.

A espécie Siparuna guianensis, conhecida vulgarmente como capitiú, foi citada no levamento etnobotânico de plantas medicinais realizado por Silva (2002) em uma comunidade quilombola, no município de Macapá-AP. As folhas desta planta são empregadas para gripe, reumatismo e inflamações.
Fachim \& Guarim (1995) alertam para o risco de extinção de algumas espécies medicinais pela falta de manejo adequado e uso indiscriminado dessas plantas, que são retiradas do seu ambiente natural, muitas vezes, inteiras e sem completar o ciclo reprodutivo, para serem comercializadas, acarretando na maioria dos casos seu desaparecimento no local de coleta.

Dentre as espécies que podem ser usadas como artesanato, Licania lata (16 ind./ha) e Clusia amazonica (16 ind./ha) apresentaram $81 \%$ da densidade total do grupo, seguidas por Licania canescens e Trichilia lecointei com respectivamente, 6 e 1 ind./ha. Das 95 espécies catalogadas por Rocha e Silva (2002), cerca de $11 \%$ possuíam como um dos seus usos o artesanato, no entanto, nenhuma das espécies encontradas no presente levantamento foram citadas.

No grupo de uso de extrativos, uma única espécie foi inventariada, Hymenaea parvifolia, apresentando um total de 4 indivíduos por hectare. Esta espécie, no estudo realizado por Vieira et al. (1996), também foi citada como de potencial resinífero.

Neste estudo, as espécies Mabea angustifolia (3 ind/ha) e Neea sp. (161 ind./ha), não foram incluídas em nenhum grupo de uso, por terem seu uso desconhecido. Entretanto, Vieira $e t$ al. (1996) citaram Mabea angustifolia e Neea sp. como espécies produtoras de lenha e carvão.

Algumas famílias foram características de determinados tipos de uso, como por exemplo, a família Lecythidaceae. Das seis espécies encontradas no levantamento, cinco pertenciam ao grupo de uso das madeiras de alto valor comercial. Outro exemplo que pode ser dado, é a família Bignoniaceae. Representada por duas espécies do gênero Tabebuia, foi exclusiva da categoria de uso acima citada.

No presente levantamento, a família Leguminosae engloba cerca de $50 \%$ do total das espécies madeireiras de alto valor comercial. Silva et al. (1986), ao estudar os aspectos florísticos da mata do aeroporto de Serra Norte-PA, afirmam que em todos os inventários realizados na região de Carajás, até o momento do seu levantamento, a família Leguminosae apresentou o maior número de espécies amostradas com representantes em todas as classes de tamanho, além de possuir uma gama muito grande de aplicabilidade econômica entre as espécies, englobando espécies madeireiras de alta qualidade, medicinais e de frutos comestíveis.

Entre as espécies utilizadas em construções rurais, as famílias Myrtaceae e Flacourtiaceae tiveram bastante destaque, obtendo cerca de $86 \%$ e $83 \%$, respectivamente, das suas espécies, classificadas nesse grupo.

\section{CONCLUSÃO}

A floresta secundária de 1,5 ha e 30 anos de idade apresentou um total de 103 espécies arbóreas, sendo a maioria utilizada nas construções rural e apontada como madeireiras de alto valor comercial.

A potencialidade de uso diversificado da floresta secundária 


\section{ACTA \\ AMAZONICA}

POTENCIAL DE USO DAS ESPÉCIES ARBÓREAS DE UMA

FLORESTA SECUNDÁRIA, NA ZONA BRAGANTINA, PARÁ, BRASIL estudada é grande. Levando-se em consideração que a composição florística da capoeira estudada não difere muito de outras da região, pode-se extrapolar este elevado potencial para as demais áreas existentes atualmente na Amazônia oriental, tornando-se um cenário animador principalmente ao agricultor familiar, e sem dúvida, um estímulo ao investimento e à conservação das florestas secundárias como um todo. Todavia, os agricultores não têm aproveitado esse potencial, provavelmente pela falta de conhecimento e de recursos para sua exploração.

Desta forma, são necessários estudos mais aprofundados na área de tecnologia e aproveitamento dos produtos oriundos de florestas secundárias, para que estas espécies sejam manejadas de forma adequada, de acordo com os seus potenciais de utilização.

\section{BIBLIOGRAFIA CITADA}

Alencar, A.; Vieira, I.C.G.; Nepstad, D.C.; Lefebvre, P. 1996. Análise multitemporal do uso da terra e mudança de vegetação em antiga área agrícola da Amazônia. In: Anais do VIII Simpósio Internacional de Sensoriamento Remoto, Salvador, Bahia. p.4.

Cavalcante, P.B. 1979. Frutas comestíveis da Amazônia, $3^{\mathrm{a}}$ ed. Belém, Museu Paraense Emílio Goeldi. 62 p.

Clement, C.R.; Arkcoll, D.B. 1979. A política florestal e o futuro promissor da fruticultura na Amazônia. Acta Amazonica, 9 (4): 173-177.

Denich, M. 1986. A vegetação da Amazônia oriental com ênfase na vegetação antrópica. Belém, EMBRAPA-CPATU. Documentos, 40: 43-69.

Denich, M. 1991. Estudo da importância de uma vegetação secundária nova para o incremento da produtividade do sistema de produção na Amazônia Oriental brasileira. Tese de Doutorado, Universidade Georg August de Göttingen, Eschborn-Alemanha. 284p.

Fachim, E.; Guarim, V.L.M. S. 1995.Conservação da biodiversidade de espécies da flora de Mato Grosso. Acta Bot. Bras., 9 (2): 281-287.

Ferreira, M.R.C. 2000. Identificação e valorização das plantas medicinais de uma comunidade pesqueira do litoral paraense (Amazônia Brasileira). Tese de Doutorado, Universidade Federal do Pará. Belém, Pará. 260p.

Finegam, B. 1992. The Management potencial of neotropical secondary lowland rain forest. For. Ecol. Manag., 47: 295321.

IBDF (Instituto Brasileiro de Desenvolvimento Florestal). 1983. Potencial Madeireiro do Grande Carajás. Brasília, 134 p.

IBGE (Instituto Brasileiro de Geografia e Estatística). 1983.
Bragança, Norte, Pará. Coleção de Monografias Municipais Nova Série. 17:1-16.

Lisboa, P.L.B. 1989. Estudo florístico da vegetação arbórea de uma floresta secundária, em Rondônia. Bol. Mus. Para. Emílio Goeldi, sér. Bot. 5(2):145-162.

Nepstad, D.C.; UHL, C.; Pereira, C.A., Silva, J.M.C . 1996. A comparative study of tree establishment in abandoned pasture and mature forest of eastern Amazonia. Oikos, 76:25-39.

Pereira, C.A.; Vieira, I.C.G. 2001. A importância das florestas secundárias e os impactos de sua substituição por plantios mecanizados de grãos na Amazônia. Interciência, 26(8):337341.

Rios, M.; Martins-da-Silva, R.C.V.; Sabogal, C.; Martins, J.; Silva, R.N.; Brito, R.R.; Brito, I.M.; Brito, M.F.C.; Silva, J.R.; Ribeiro, R.T. 2001. Benefícios das plantas da capoeira para a comunidade de Benjamin Constant, Pará, Amazônia Brasileira. Belém, CIFOR. 54 p.

Rocha, A.E.S.; Silva, M.F.F. 2002. Catálogo de espécies de floresta secundária. Belém, Museu Paraense Emílio Goeldi, 212p.

Shanley, P.; Cymeriys, M.; Galvão, J. 1998. Frutíferas da mata na vida amazônica. Editora Supercores, Belém. 127p.

Silva, M.F.F.; Rosa, N.A.; Salomão, R.P. 1986. Estudos botânicos na área do Projeto Ferro Carajás. 3. Aspectos florísticos da Mata do Aeroporto de Serra de Norte-PA. Bol. Mus. Para. Emílio Goeldi, sér. Bot. 2(2):169-187.

Silva, R.B.L. 2002. A etnobotânica de plantas medicinais da comunidade quilombola de Curiaú, Macapá-AP, Brasil. Dissertação de Mestrado, Faculdade de Ciências Agrárias do Pará. Belém, Pará. 170p.

Viera, I.C.G. 1996. Forest succession after shifting cultivation in Eastern Amazonia. Tese de Doutorado, Universidade de Stirling, Scotland. 215p.

Vieira, I.C.G.; Galvão, N.; Rosa, N.A. 1996 . Caracterização morfológica de frutos e germinação de sementes de espécies arbóreas nativas da Amazônia. Bol. Mus. Para. Emílio Goeldi, sér. Bot. 12(2): 271-288.

Vieira, L.S. 1991. Manual da medicina popular: a fitoterapia da Amazônia. Faculdade de Ciências Agrárias do Pará. Belém. 248p.
RECEBIDO EM 28/04/2004
ACEITO EM 01/11/2005 
Anexo 1 - Nome científico, densidade (número de indivíduos/ha) e área basal $\left(\mathrm{m}^{2} / \mathrm{ha}\right)$ por grupo de uso e família das espécies encontradas em uma floresta secundária, no município de Bragança, PA.

\begin{tabular}{|c|c|c|c|}
\hline Grupo de uso/ Família & Nome científico & Densidade & Área basal \\
\hline \multicolumn{4}{|c|}{ Madeira de Alto Valor Comercial } \\
\hline \multirow[t]{2}{*}{ BIGNONIACEAE } & Tabebuia ochracea (Cham.) Standl. & 0,67 & 0,011 \\
\hline & Tabebuia serratifolia (Vohl) G. Nicholson & 2,00 & 0,007 \\
\hline BOMBACACEAE & Eriotheca globosa (Aubl.) A. Robyns & 0,67 & 0,008 \\
\hline CARYOCARACEAE & Caryocar villosum (Aubl.) Pers. & 1,33 & 0,013 \\
\hline COMBRETACEAE & Terminalia amazonia (J. F. Gmel.) Exell & 1,33 & 0,038 \\
\hline \multirow[t]{2}{*}{ EUPHORBIACEAE } & Croton matourensis Aubl. & 57,33 & 1,511 \\
\hline & Drypetes variabilis Uittiem & 0,67 & 0,005 \\
\hline GUTTIFERAE & Rheedia acuminata (Ruiz \& Pav.) Planch. \& Triana & 3,33 & 0,020 \\
\hline HUMIRIACEAE & Sacoglottis amazonica Mart. & 129,33 & 1,077 \\
\hline \multirow[t]{2}{*}{ LAURACEAE } & Licaria canella (Meissner) Kosterm. & 4,67 & 0,030 \\
\hline & Ocotea guianensis Aubl. & 2,00 & 0,024 \\
\hline \multirow[t]{5}{*}{ LECYTHIDACEAE } & Couratari guianensis Aubl. & 1,33 & 0,005 \\
\hline & Couratari oblongifolia Ducke \& Knuth & 14,67 & 0,063 \\
\hline & Eschweilera coriacea (DC.) S.A. Mori & 76,67 & 0,336 \\
\hline & Lecythis lurida (Miers) S.A. Mori & 48 & 0,236 \\
\hline & Lecythis pisonis Cambers. & 22,00 & 0,107 \\
\hline \multirow[t]{11}{*}{ LEGUMINOSAE } & Abarema cochleata (Willd.) Barneby \& J. W. Grimes & 3,33 & 0,143 \\
\hline & Bowdichia nitida Spruce ex Benth. & 0,67 & 0,002 \\
\hline & Diplotropis guianensis Benth. & 6,00 & 0,089 \\
\hline & Diplotropis purpurea (Rich.) Amshoff & 0,67 & 0,003 \\
\hline & Dipteryx odorata (Aubl.)Willd. & 8,67 & 0,087 \\
\hline & Enterolobium schomburgkii (Benth.) Benth. & 8,67 & 0,205 \\
\hline & Hymenaea courbaril L. & 2,67 & 0,015 \\
\hline & Inga alba (Sw.) Willd. & 8,67 & 0,087 \\
\hline & Ormosia flava (Ducke) Rudd & 118,67 & 1,129 \\
\hline & Schizolobium amazonicum Huber ex Ducke & 0,67 & 0,010 \\
\hline & Sclerolobium chrysophyllum Poepp. & 8,67 & 0,311 \\
\hline MORACEAE & Brosimum guianense (Aubl.) Huber & 0,67 & 0,002 \\
\hline RUTACEAE & Zanthoxylum rhoifolium Lam. & 1,33 & 0,004 \\
\hline \multirow[t]{2}{*}{ SAPOTACEAE } & Franchetella gongrijpii (Eyma) Aubrev. & 1,33 & 0,005 \\
\hline & Pouteria guianensis Aubl. & 0,67 & 0,003 \\
\hline \multicolumn{4}{|c|}{ Madeira de Baixo Valor Comercial } \\
\hline$\overline{\text { ANACARDIACEAE }}$ & Tapirira guianensis Aubl. & 46,00 & 1,097 \\
\hline \multirow[t]{2}{*}{ CHRYSOBALANACEAE } & Couepia bracteosa Benth. & 0,67 & 0,002 \\
\hline & Licania densiflora Kleinhoonte & 12,00 & 0,102 \\
\hline DICHAPETALACEAE & Tapura amazonica Poepp. & 2,00 & 0,008 \\
\hline EUPHORBIACEAE & Conceveiba guianensis Aubl. & 1,33 & 0,005 \\
\hline \multirow[t]{2}{*}{ LEGUMINOSAE } & Ormosia paraensis Ducke & 0,67 & 0,002 \\
\hline & Sclerolobium paraense Huber & 4,67 & 0,178 \\
\hline MYRISTICACEAE & Iryanthera juruensis Warb. & 0,67 & 0,007 \\
\hline MYRTACEAE & Marlierea sp. & 1,33 & 0,005 \\
\hline \multicolumn{4}{|c|}{ Madeira para Construção Rural } \\
\hline ANACARDIACEAE & Thyrsodium paraense Huber & 7,33 & 0,049 \\
\hline ANNONACEAE & Guatteria poeppigiana Mart. & 26,00 & 0,157 \\
\hline BOMBACACEAE & Pachira faroensis (Ducke) W.S. Alverson & 2,00 & 0,011 \\
\hline
\end{tabular}




\section{ACTA AMAZONICA}

\begin{tabular}{|c|c|c|c|}
\hline Grupo de uso/ Família & Nome científico & Densidade & Área basal \\
\hline CONNARACEAE & Connarus perrottetii (DC.) Planch. & 10,67 & 0,60 \\
\hline EBENACEAE & Diospyros sp. & 0,67 & 0,004 \\
\hline \multirow[t]{3}{*}{ EUPHORBIACEAE } & Maprounea guianensis Aubl. & 158,00 & 1,612 \\
\hline & Pogonophora schomburgkiana Miers ex Benth. & 38,00 & 0,149 \\
\hline & Phyllanthus nobilis (L. f.) Müll. Arg. & 87,33 & 1,062 \\
\hline ELAOCARPACEAE & Sloanea froesii Earle Sm. & 52,67 & 0,324 \\
\hline \multirow[t]{5}{*}{ FLACOURTIACEAE } & Casearia aculeata Jacq. & 3,33 & 0,008 \\
\hline & Casearia arborea (Rich.) Urb. & 88,00 & 0,375 \\
\hline & Casearia decandra Jacq. & 3,33 & 0,009 \\
\hline & Casearia javitensis Kunth & 2,00 & 0,009 \\
\hline & Lindackeria paraensis Kuhlm. & 0,67 & 0,002 \\
\hline GUTTIFERAE & Vismia guianensis (Aubl.) Pers. & 0,67 & 0,013 \\
\hline LACISTEMACEAE & Lacistema aggregatum (P.J Bergius) Rusby & 10,00 & 0,035 \\
\hline LECYTHIDACEAE & Eschweilera grandiflora (Aubl.) Sandwith & 3,33 & 0,018 \\
\hline LEGUMINOSAE & Swartzia arborescens (Aubl.) Pittier & 3,33 & 0,017 \\
\hline \multirow[t]{3}{*}{ MALPIGHIACEAE } & Byrsonima crispa A. Juss. & 1,33 & 0,087 \\
\hline & Byrsonima densa (Poir.) DC. & 11,33 & 0,211 \\
\hline & Byrsonima chrsophylla Kunth & 37,33 & 1,191 \\
\hline MELASTOMATACEAE & Bellucia grossularioides (L.) Triana & 2,67 & 0,009 \\
\hline \multirow[t]{7}{*}{ MYRTACEAE } & Eugenia biflora (L.) DC. & 4,67 & 0,012 \\
\hline & Eugenia paraensis O. Berg & 1,33 & 0,008 \\
\hline & Myrcia atramentifera Barb. Rodr. & 1,33 & 0,005 \\
\hline & Myrcia bracteata (Rich.) DC. & 92,00 & 0,509 \\
\hline & Myrciaria sp. & 20,67 & 0,048 \\
\hline & Myrciaria tenella (DC.) O. Berg & 126,67 & 0,386 \\
\hline & Psidium guianensis Sw. & 3,33 & 0,008 \\
\hline OCHNACEAE & Ouratea castaneifolia (DC.) Engl. & 50,00 & 0,335 \\
\hline RUTACEAE & Pilocarpus sp. & 4,00 & 0,010 \\
\hline \multirow[t]{3}{*}{ SAPINDACEAE } & Sapindus saponaria L. & 10,00 & 0,046 \\
\hline & Talisia guianensis Aubl. & 5,33 & 0,016 \\
\hline & Talisia longifolia (Benth.) Radlk. & 55,33 & 0,257 \\
\hline SAPOTACEAE & Richardella macrophylla (Lam.) Aubrév. & 1,33 & 0,003 \\
\hline \multicolumn{4}{|l|}{ Madeira para Lenha } \\
\hline ANNONACEAE & Xylopia aromatica (Lam.) Mart. & 3,33 & 0,015 \\
\hline FLACOURTIACEAE & Banara guianensis Aubl. & 0,67 & 0,005 \\
\hline \multirow[t]{6}{*}{ LEGUMINOSAE } & Abarema jupunba (Willd.) Britton \& Killip & 39,33 & 0,166 \\
\hline & Inga heterophylla Willd. & 3,33 & 0,013 \\
\hline & Inga nitida Willd. & 2,67 & 0,007 \\
\hline & Inga paraensis Ducke & 6,67 & 0,027 \\
\hline & Inga rubiginosa (Rich.) DC. & 12,67 & 0,138 \\
\hline & Stryphnodendron guianense (Aubl.) Benth. & 5,33 & 0,082 \\
\hline OLACACEAE & Chaunochiton kappleri (Sagot ex Engl.) Ducke & 2,00 & 0,036 \\
\hline \multicolumn{4}{|l|}{ Frutos } \\
\hline ANNONACEAE & Annona paludosa Aubl. & 71,33 & 0,796 \\
\hline GUTTIFERAE & Platonia insignis Mart. & 24,00 & 0,272 \\
\hline \multirow[t]{3}{*}{ LEGUMINOSAE } & Inga edulis Mart. & 5,33 & 0,032 \\
\hline & Inga macrophylla Bonpl. ex Willd. & 2,00 & 0,006 \\
\hline & Inga nobilis Willd. & 5,33 & 0,020 \\
\hline
\end{tabular}




\section{ACTA \\ AMAZONICA}

POTENCIAL DE USO DAS ESPÉCIES ARBÓREAS DE UMA

FLORESTA SECUNDÁRIA, NA ZONA BRAGANTINA, PARÁ, BRASIL

\begin{tabular}{|c|c|c|c|}
\hline Grupo de uso/ Família & Nome científico & Densidade & Área basal \\
\hline MALPIGHIACEAE & Byrsonima aerugo Sagot & 2,00 & 0,026 \\
\hline MYRTACEAE & Psidium guajava L. & 12,00 & 0,033 \\
\hline \multicolumn{4}{|l|}{ Extrativos } \\
\hline LEGUMINOSAE & Hymenaea parvifolia Huber & 4,00 & 0,015 \\
\hline \multicolumn{4}{|l|}{ Medicinal } \\
\hline APOCYNACEAE & Himatanthus sucuuba (Spruce ex Müll. Arg.) Woodson & 20,67 & 0,226 \\
\hline LEGUMINOSAE & Stryphnodendron adstringens (Mart.) Coville & 0,67 & 0,002 \\
\hline MONIMIACEAE & Siparuna guianensis Aubl. & 0,67 & 0,001 \\
\hline THEOPHRASTACEAE & Clavija lancifolia Desf. & 0,67 & 0,014 \\
\hline TILIACEAE & Luehea sp. & 0,67 & 0,007 \\
\hline \multicolumn{4}{|l|}{ Artesanal } \\
\hline \multirow[t]{2}{*}{ CHRYSOBALANACEAE } & Licania canescens Benoist & 6,00 & 0,039 \\
\hline & Licania lata J. F. Macbr. & 16,00 & 0,099 \\
\hline GUTTIFERAE & Clusia amazonica Planch. \& Triana & 16,00 & 0,069 \\
\hline MELIACEAE & Trichilia lecointei Ducke & 1,33 & 0,009 \\
\hline \multicolumn{4}{|l|}{ Uso não Conhecido } \\
\hline EUPHORBIACEAE & Mabea angustifolia Spruce ex Benth. & 3,33 & 0,010 \\
\hline NYCTAGINACEAE & Neea sp. & 160,67 & 0,930 \\
\hline
\end{tabular}

